\title{
DeVElopMent of 2.8-GHz Solar Flux ReCEIVERS
}

\author{
Younguoo Yun ${ }^{1}$, Yong-Sun Park ${ }^{2}$, Chang-Hee Kim² ${ }^{2}$, Bangwon LeE ${ }^{2}$, Jung-Hoon Kim ${ }^{3}$, SAeho Yoo ${ }^{3}$, \\ Chul-Hwan LeE ${ }^{3}$, Jinwook $\mathrm{HAN}^{4}$, And Young Yun Kim ${ }^{4}$ \\ ${ }^{1}$ Korea Astronomy and Space Science Institute, Daejeon 305-348, Korea; yjyun@kasi.re.kr \\ ${ }^{2}$ Department of Physics and Astronomy, Seoul National University, Seoul 151-742, Korea \\ ${ }^{3}$ Space and Earth Technology System, Seoul 137-130, Korea \\ ${ }^{4}$ Korean Space Weather Center, National Radio Research Agency, Jeju 695-922, Korea
}

Received May 8, 2014; accepted June 24, 2014

\begin{abstract}
We report the development of solar flux receivers operating at $2.8 \mathrm{GHz}$ to monitor solar radio activity. Radio waves from the sun are amplified, filtered, and then transmitted to a power meter sensor without frequency down-conversion. To measure solar flux, a calibration scheme is designed with a noise source, an ambient load, and a hot load at $100{ }^{\circ} \mathrm{C}$. The receiver is attached to a $1.8 \mathrm{~m}$ parabolic antenna in Icheon, owned by National Radio Research Agency, and observation is being conducted during day time on a daily basis. We compare the solar fluxes measured for last seven months with solar fluxes obtained by DRAO in Penticton, Canada, and by the Hiraiso solar observatory in Japan, and finally establish equations to convert observed flux to the so-called Penticton flux with an accuracy better than 3.2 sfu.
\end{abstract}

Key words: instrumentation: miscellaneous — methods: observational — Sun: activity — Sun: radio radiation

\section{INTRODUCTION}

Modern human life relies largely on wireless communication devices, such as mobile phones. These devices continue to become smaller and consume progressively less power for mobility reasons. As a result, they are subjected to more environmental disturbances, one of which is solar radio activity. There have been reports that satellites and wireless devices on the ground can be severely affected by solar activity. Ground based GPS receivers, for example, experience carrier-to-noise ratio degradation due to large solar radio bursts (Cerruti et al. 2006). Wireless communication systems such as cellular phones, can also experience interference and interruption of service (Gary et al. 2005).

For this reason, it is important to monitor solar activity in the radio range. The $2.8 \mathrm{GHz}$ or $10.7 \mathrm{~cm}$ solar radio flux has long been monitored as a representative indicator of solar radio activity since 1946, currently by Dominion Radio Astrophysical Observatory in Penticton, Canada (Tapping \& Charrois 1994; Tapping \& Morton 2013). The Penticton data have since become the reference that other observatories or instrumentations are compared to, because of the careful calibration and the widest time coverage. Observations in other frequencies are often scaled to the 10.7 cm flux (Tapping 2001). However there is a time lag of seven hours between Penticton and Korea and the observatory releases the data that are collected only three times a day around local noon. The observatory aims to monitor long term solar activity. On the other hand, Hiraiso solar observatory in Japan presents large number of solar fluxes taken during daytime, but access to them is delayed by at least a day. Therefore, it is

CORRESPONDING AUthoR: Y.-S. Park necessary to establish a national monitoring system of solar radio activity independently so that the government can promptly respond to strong radio bursts that may severely damage the country's telecommunication system.

In order to monitor solar activity, we have designed and built receivers that can measure solar radio flux on an absolute scale every day from sunrise to sunset to an accuracy of $\lesssim 5 \%$. If the receivers are properly calibrated, they could also be used to monitor the activity on a long solar cycle scale. In Section 2, we present the design and fabrication of the system. Sections 3 and 4 describe the calibration procedure and software development, respectively. In Sections 5 and 6, we discuss observation results and the final calibration procedure, respectively.

\section{DESIGN AND FABRICATION}

\subsection{General}

The receiver should be able to measure the absolute solar radio intensity with the appropriate calibration devices and scheme. Calibration is usually conducted with radio sources of known intensity, which, in this work, are a noise source with known power and termination loads (Wilson et al. 2009). In addition, the system must be linear over a wide input range. Considering these conditions, we have designed a radio receiver as shown in Figure 1. The signal from the feed is amplified, filtered, and detected without frequency down-conversion. A conventional design with mixers and local oscillators may be more economical, but a system without down-conversion is simpler and provides linearity over a wider input power range.

An RF switch selects signals from either the Sun or 




Figure 1. Block diagram of the $2.8 \mathrm{GHz}$ receiver

the calibrators. The noise source is turned on and off by a $5 \mathrm{~V}$ TTL. The calibration noise source should be as close as possible to the feed. For ease of fabrication, in this work we have placed the noise source close to the input port of the receiver. Because there is a four meter-long cable between the receiver and the feed cable loss compensation is necessary, which is discussed in Section 3. The first amplifier is a low noise amplifier with a noise figure of $N F=0.4 \mathrm{~dB}$. To avoid any radio frequency interference, the radio environment was monitored and narrow band-pass filters were used with a final bandwidth of $20 \mathrm{MHz}$ centered at $2.8 \mathrm{GHz}$. The total amplification was $100 \mathrm{~dB}$. In the original design in 2008 , the power detection part consisted of a square law detector and a voltage-to-frequency converter. Since recent technological advances have made a power meter sensor with a wider dynamic range available at low cost, we replaced the original power detection part with a power meter sensor.

\subsection{Noise Source and Temperature Sensor}

The power of the noise source is typically $E N R=32$ $\mathrm{dB}$, or $T_{n}=4.6 \times 10^{5} \mathrm{~K}$, which is significantly higher than the typical solar radio flux. A directional coupler attenuates the noise power by $\sim 20 \mathrm{~dB}$ before reaching the RF switch. The directional coupler passes the power of the ambient load with little attenuation, as shown in Figure 1. The output of the ambient load is directly proportional to the ambient temperature. The power of the noise source depends weakly on the ambient temperature. Thus, monitoring the ambient temperature is necessary and it is performed by two temperature sensors attached to the ambient load and the noise source. The temperature readout, and the control of the RF switch and the noise source are carried out by a short, low-level program installed on a microcontroller unit (MCU). Computer programs record the measured data from the power sensor via a USB interface and the temperature information from the MCU via an RS-232 interface.

\subsection{Fabrication}

We have fabricated two receiver units, referred to as $\mathrm{Rx} 1$ and $\mathrm{Rx} 2$ hereafter. One of the receivers is shown in Figure 2, the dimensions of which are $494 \mathrm{~mm}(\mathrm{~L}) \times 334$ $\mathrm{mm}(\mathrm{W}) \times 92 \mathrm{~mm}(\mathrm{H})$. One can see the input port for signals from the sun in the lower left corner, and the MCU and lines from/to the PC in the upper left. The power supply is found on the right. The two receivers are attached to an antenna that is located in Icheon and managed by National Radio Research Agency (RRA) in Korea. It is a parabolic antenna with diameter of 1.8 m, fabricated by High Gain Antenna, Korea. It is of the alt-azimuth type with $\mathrm{f} / \mathrm{D}$ ratio of 0.4 . The receivers are mounted on the rear of the parabolic dish. The beam size of the antenna is $4.2^{\circ}$ and therefore encompasses the whole solar disc. A feed horn at prime focus has two output ports with mutually orthogonal linear polarization. Usually a hybrid coupler is attached to these ports, to yield signals of left and right circular polarization. In this study, however, the two linear polarization ports are directly connected to the two receivers, individually. The degree of polarization, averaged over the solar disc, should be negligible (Tapping 2001). One of the two receivers could be treated as a backup of the other. We can also test the calibration accuracy with the two receivers as mentioned in Section 6 .

\section{Calibration}

\subsection{Formulation}

Since we want to measure the absolute flux of the sun, calibration plays a very important part. The system must be linear over a wide input range, and an appropriate calibration scheme should be designed. At the heart of the calibration is the relationship between the solar flux and the output, which is usually a digital number (Wilson et al. 2009).

When the telescope points to the sun, the output 


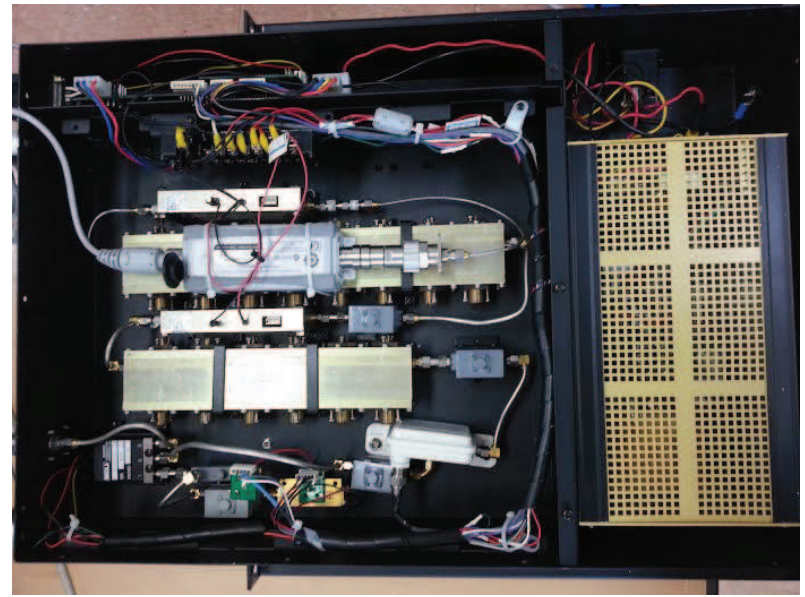

Figure 2. Inner view of the receiver with a cover removed

power, $P_{\text {sun }}$ can be written as

$$
\begin{aligned}
P_{\text {sun }} & =G\left[L \left\{\eta \left(\left(A_{e} / 2 k\right) S e^{-\tau}\right.\right.\right. \\
& \left.\left.+T_{a t m}\left(1-e^{-\tau}\right)\right)+(1-\eta) T_{a m b}\right\} \\
& \left.+(1-L) T_{\text {cable }}+T_{r x}\right],
\end{aligned}
$$

where $A_{e}=\eta_{A} \pi D^{2} / 4$ is an effective collecting area, $k$ is Boltzmann's constant, $\eta$ is the telescope's loss, and $\tau$ is the atmospheric optical depth. The $T_{a t m}, T_{a m b}$, and $T_{\text {cable }}$ correspond to atmospheric temperature, ambient temperature, and the temperature of the cable from the feed to the receiver, respectively. $T_{r x}$ is the receiver noise temperature, measured in front of the RF switch. $S$ is the solar flux above the atmosphere, which is what we want to measure. $G$ includes amplification factors, losses, and a factor for the analog to digital conversion at the receiver. $L$ is the cable loss. When the telescope looks at the sky,

$$
\begin{aligned}
P_{\text {sky }} & =G[L\{\eta( \\
& \left.\left.+T_{a t m}\left(1-e^{-\tau}\right)\right)+(1-\eta) T_{a m b}\right\} \\
& \left.+(1-L) T_{\text {cable }}+T_{r x}\right] .
\end{aligned}
$$

The difference between the two equations yields an expression in which some unknown terms cancel

$$
P_{\text {sun }}-P_{\text {sky }}=G \operatorname{Ln}\left(A_{e} / 2 k\right) S e^{-\tau} .
$$

To evaluate $G$, we measure powers $P_{n}$ and $P_{a m b}$ for the two inputs, the noise source and the ambient load. We then use the following expression for $G$,

$$
G=\left(P_{n}-P_{a m b}\right) /\left(T_{n}-T_{a m b}\right) .
$$

Finally, an expression for the solar flux, $S$, is derived as

$$
S=\frac{\left(P_{s u n}-P_{s k y}\right)}{\left(P_{n}-P_{a m b}\right)}\left(T_{n}-T_{a m b}\right) \frac{2 k e^{\tau}}{L \eta A_{e}} .
$$

The cable loss, $L$, is estimated to be 0.79 , and the antenna loss, $\eta$, is known to be close to 0.9 . The atmosphere is almost transparent at $2.8 \mathrm{GHz}$, and we set $\tau=0.025$, following Tapping (2001).
To measure the effective antenna collecting area, $A_{e}$, a reference antenna, which is usually a pyramidal horn, may be used (Tapping 2001). Since the antenna parameters are theoretically well known for the pyramidal horn, one can get $A_{e}$, by measuring and comparing the output powers from the reference antenna and the parabolic antenna toward a common radio source. We have tried this method, but the tracking of the horn antenna was not accurate and the measurement introduced additional unknown variables. Therefore we have assigned a typical value of 0.55 to the aperture efficiency of the antenna. Consequently, the calibration scheme developed is a process to obtain only partially the absolute solar flux, since some variables have not been measured directly. An additional process to convert the measured flux to the absolute flux is necessary, which is discussed in Section 6 .

\subsection{Measurement of Noise Power}

Though the manufacturer specifies the power of the noise source to be $E N R\left(\right.$ at $\left.24^{\circ} \mathrm{C}\right)=32.5$ and $31.3 \mathrm{~dB}$ for $\mathrm{Rx} 1$ and $\mathrm{Rx} 2$, respectively, it experiences attenuation before reaching the $\mathrm{RF}$ switch due to the directional coupler and cables, which amounts to $\sim 20 \mathrm{~dB}$ with $\sim 0.3 \mathrm{~dB}$ uncertainty. We have, therefore, measured the exact noise temperature, $T_{n}$ in front of the RF switch by applying a method similar to the one described above. Having measured the outputs of the receiver, $P_{a m b}, P_{h o t}$, and $P_{n}$, when the input is an ambient load at room temperature $T_{a m b}$, an ambient load at $T_{h o t}=373 \mathrm{~K}$ (boiling water), and a noise source, respectively, we obtain the following equation for $T_{n}$,

$$
T_{n}=\frac{\left(P_{n}-P_{h o t}\right)}{\left(P_{n}-P_{a m b}\right)}\left(T_{n}-T_{a m b}\right)+T_{h o t} .
$$

It is found that $T_{n}=6000 \mathrm{~K}$ and $4800 \mathrm{~K}$, respectively, for the two receivers, $\mathrm{Rx} 1$ and $\mathrm{Rx} 2$ at $T_{a m b}=24^{\circ} \mathrm{C}$. The noise source's power depends very weakly on ambient temperature as follows

$$
\operatorname{ENR}(T)=\operatorname{ENR}\left(\text { at } 24^{\circ} C\right)+0.01 \times\left(T_{a m b}-24\right),
$$

where $T_{a m b}$ is the ambient temperature in ${ }^{\circ} \mathrm{C}$. Here, the coefficient $0.01 \mathrm{~dB} /{ }^{\circ} \mathrm{C}$ is provided by the maker and is therefore not confirmed, which may lead to calibration errors.

\section{Software}

\subsection{General}

The software consists of three parts: the antenna control program, the receiver control program, and the data reduction program. The antenna control program has been coded with LabVIEW. The receiver control program is written in $\mathrm{C}$, and the data reduction program is written in Python. Each program has been developed independently and operates on a different computer.

The main function of the antenna control program is to make the antenna track the sun with high precision. In order to achieve the required accuracy, the 

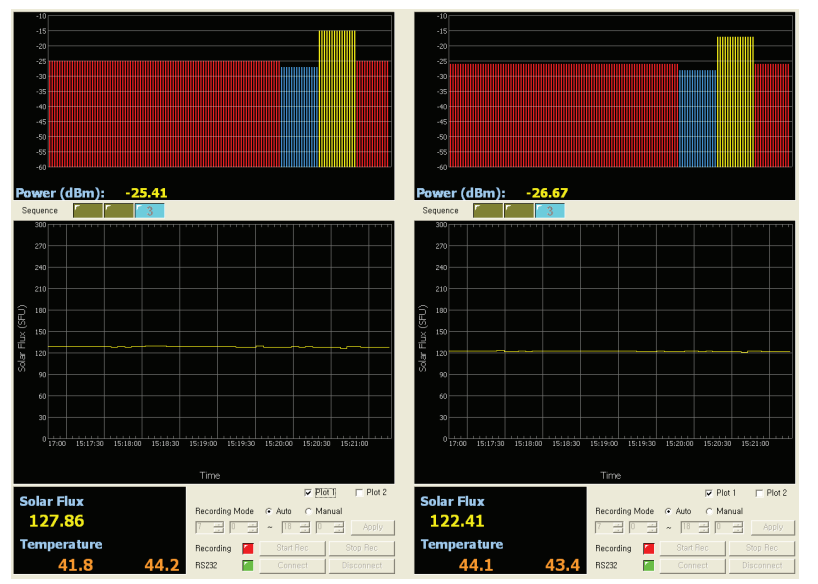

Figure 3. Graphical user interface of the program installed on the local PC. The data sets obtained by the two receivers are displayed separately in the left and right panels. The two top panels show the measured powers, in which blue, yellow, and red verical bars represent the powers of ambient load, noise source, and sun, respectively.

control program uses time information obtained from a GPS to calculate the apparent position of the sun. This program finally sends the sun's position to the antenna drive controller to accurately follow the sun.

The receiver control program consists of two parts, one of which is installed on the microcontroller unit in the receiver, and the other on a local $\mathrm{PC}$. The MCU program assigns the control functions to the $\mathrm{I} / \mathrm{O}$ ports of the MCU, and these ports are directly connected to components of the receiver such as the RF switch, the noise source, and the temperature sensors. The MCU also possesses an RS-232 interface to communicate with an external device, the local PC in this work. The program on the $\mathrm{PC}$ manages the whole observation process by communicating with the MCU. All control commands are sent from the PC to the MCU, and the temperature readouts are sent from the MCU back to the PC via the RS-232 interface. The measured powers for the sun, the noise source, and the ambient load are all transmitted from the power sensor to the PC through the USB interface. The program also calculates the solar flux as soon as the measured powers and temperatures are transmitted to the local PC, and then saves all data on a local hard disk. All powers, temperatures, and the calculated solar fluxes are displayed on the local PC's monitor. Figure 3 demonstrates the program running on the local PC.

The data reduction program is installed on the data server of RRA's Space Weather Center and gathers locally saved raw data through TCP/IP communication. This program processes the raw data for a specific purpose and displays them on the center's webpage on a daily basis.

\subsection{Microcontroller Unit}

We employ the MCU for the RF switch selection, the noise source on/off and the temperature sensor monitoring. ATmega128, manufactured by Atmel corporation, is used for this purpose. This is an 8-bit microcontroller with $128 \mathrm{~KB}$ in-system programmable flash memory and $16 \mathrm{MHz}$ operating frequency. The source code is downloaded into the flash memory through JTAG interface, and a write/erase cycle is available for up to ten thousand times.

In the earlier (2008) version of the software, the microprocessor uses the counts generated from the voltage-to-frequency converter (VFC) as an interrupt signal that controls the observation processes. The measured VFC counts and the temperature sensor's values were transmitted periodically from the microprocessor to the local PC through the RS-232 interface. This means that the receiver functions independently of any external control and generates data for itself. The PC side program is, therefore, only responsible for receiving and managing the data from the receiver.

The power detection part, using the square law detector with the VFC, has recently been replaced by a power meter sensor and consequently the operation scheme has been modified accordingly. In place of the VFC counter, the serial communication command via RS-232 interface is used as the interrupt signal that is transmitted from the PC side program to the MCU. Therefore, the MCU waits for the external control command while being in an infinite loop. When the control command from the PC arrives at the MCU and interrupts the infinite loop, the MCU proceeds with the corresponding operation.

\section{ObServation}

The observation sequence is repeated every $170 \mathrm{~s}$. The sequence consists of three steps. Firstly, the power of the ambient load is measured 20 times for $12 \mathrm{~s}$, and then the noise source's power is measured in a similar manner. Finally, the power of the sun is measured 300 times for $145 \mathrm{~s}$. While conducting the third step, for every 15 measurements, the temperatures of the two sensors are recorded. This sequence is repeated indefinitely. Whatever the status of the receiver is, the antenna looks at off-center position displaced by $10^{\circ}$ with respect to the sun every hour for 5 minutes.

Routine observation begun in 2008, but was subsequently interrupted and resumed in July 2013 following the replacement of a power meter sensor and accompanying software revision. Tracking was checked and improved by the five-point observation of the sun. The variation in the output of the receiver due to pointing error appears to be less than 3-4\%.

Figure 4 shows typical observations toward the sun made on 29 January, 2014. A radio burst occurred at approximately 13:20 KST. Also shown is the radio frequency interference recorded at 13:10 KST, the power of which was out of range. We intermittently detect such artificial signals that probably emanate from air- 


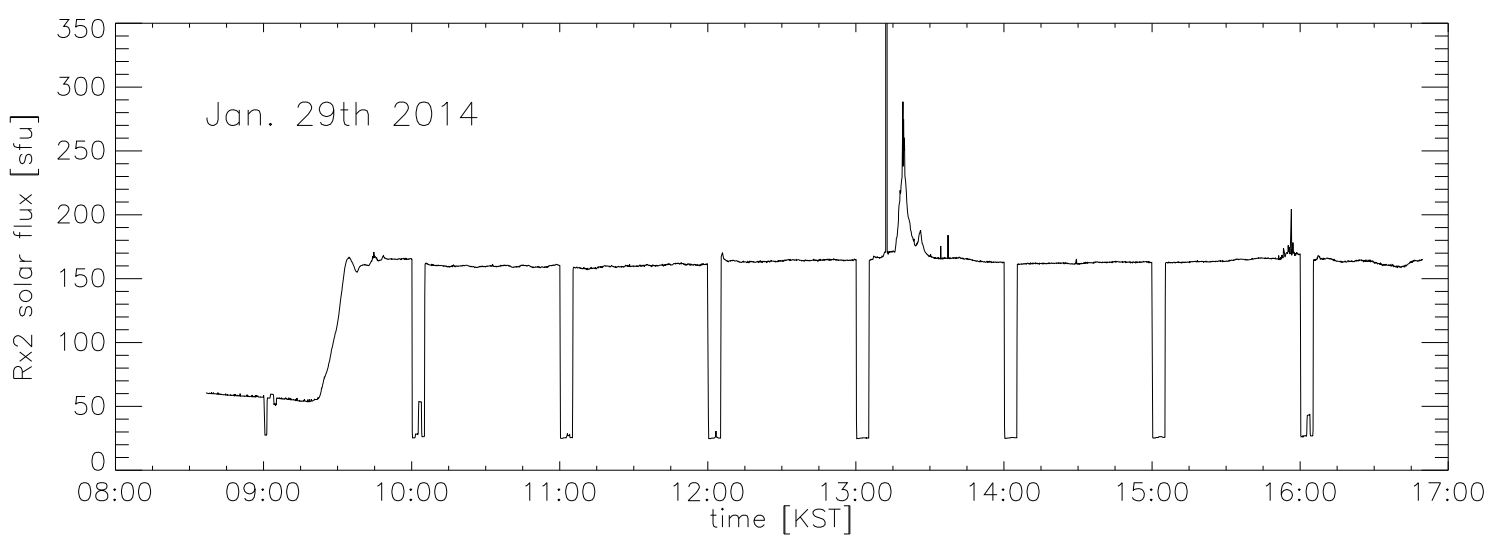

Figure 4. A sample of solar flux data taken on 29 January 2014. An outburst is noted around 13:20 KST. The out of range peak seen at about 13:10 seems to have been caused by an artificial signal. Flux values of about 30 sfu are taken every hour while the telescope points to the blank sky. 1 sfu corresponds to $10^{-22} \mathrm{Wm}^{-2} \mathrm{~Hz}^{-1}$.

planes.

\section{Comparison with Penticton and Hiraiso SOLAR FLUXES}

Even though we conducted an elaborate calibration procedure, the derived solar fluxes could have been subjected to systematic errors. To determine the accuracy of the calibration, we compared our measurements with those of Penticton, Canada and Hiraiso solar observatory, Japan. We downloaded the Penticton data from the Space Weather Canada archive ${ }^{\mathbf{1}}$, and the Hiraiso solar fluxes from the HiRAS data archive ${ }^{2}$. The Hiraiso data are calibrated with reference to the Penticton solar flux (Kubo, private communication).

Figure 5 displays the daily variation in the solar fluxes taken at local noon from 1 August, 2013 to 16 February, 2014, for three sites. We choose this time range to detect any seasonal variation in the flux from Icheon. The measurement time is the same for both Japan and Korea (03h UT), while the Canadian local noon arrives seven hours earlier. It was found that during the first three months, solar radio activity gradually decreased and then increased before suffering violent changes. Because of the time difference between Canada and the Asian countries, solar fluxes sometimes exhibit large variations. On a larger time scale, their time variations are similar. However, Hiraiso fluxes are in general larger than Penticton fluxes when the solar activity is low, whereas the trend is reversed when the solar activity is high. Icheon data is generally smaller than those of Penticton, and the difference seems to be constant, even though this relationship has become unclear during the last two months.

In order to unearth these trends, we compare the solar fluxes taken from these observatories in Figure 6 . Since we have two receiver sets, the comparison is performed for each individually. For Rx1, in the upper left frame, it was found that the Icheon data are tightly correlated with those of Hiraiso. The slope, however, is

\footnotetext{
${ }^{1}$ http://www.spaceweather.ca/solarflux/sx-eng.php

${ }^{2}$ http://sunbase.nict.go.jp/solar/denpa/index.html
}

different from unity. On the other hand, for the Rx2 in the upper right frame, slope is close to unity, but the offset is significant. In both figures, a slight upturn is noted for large solar flux. Considering the fact that our system has a rather wide dynamic range, we may conclude that the receiver of Hiraiso is saturated at larger inputs. It is also noted that the relationship depends weakly on season or air temperature. The blue crosses correspond to data taken during summer, while the red crosses during winter. The weak seasonal dependence may be attributed to the uncertain temperature dependence of the noise source. The outliers observed could be attributed to the different weather conditions at the sites. The measurements are actually affected by, e.g., heavy rain.

In the lower left panel, we compare the fluxes of Icheon Rx1 and Penticton. Contrary to the IcheonHiraiso relationship in the upper left figure, the relationship appears to be linear. However, this may be simply due to the large scatter of the data, a result of the difference in measurement times. The figure in lower right panel shows the relationship between $\mathrm{Rx} 2$ and the Penticton data; the slope is clearly smaller than that in the lower left figure. Since the Penticton data is regarded to be the norm, we derive linear equations between Icheon and Penticton fluxes as follows, using bisector least square regression (Isobe et al. 1990).

$$
S_{R x 1}=(0.99 \pm 0.01) S_{P}-(4.0 \pm 0.3)
$$

and

$$
S_{R x 2}=(0.84 \pm 0.01) S_{P}+(5.7 \pm 0.2),
$$

where $S_{R x 1}$ and $S_{R x 2}$ are Icheon fluxes and $S_{P}$ is Penticton fluxes. For the $\mathrm{Rx} 1$, the $\mathrm{y}$-intercept is small and the slope is close to unity, suggesting that the calibration was relatively accurate. For $\mathrm{Rx} 2$, however, the slope significantly deviates from one, while the y-intercept is similar. This suggests that the calibration process contains large systematic errors and that further improvement is required. 


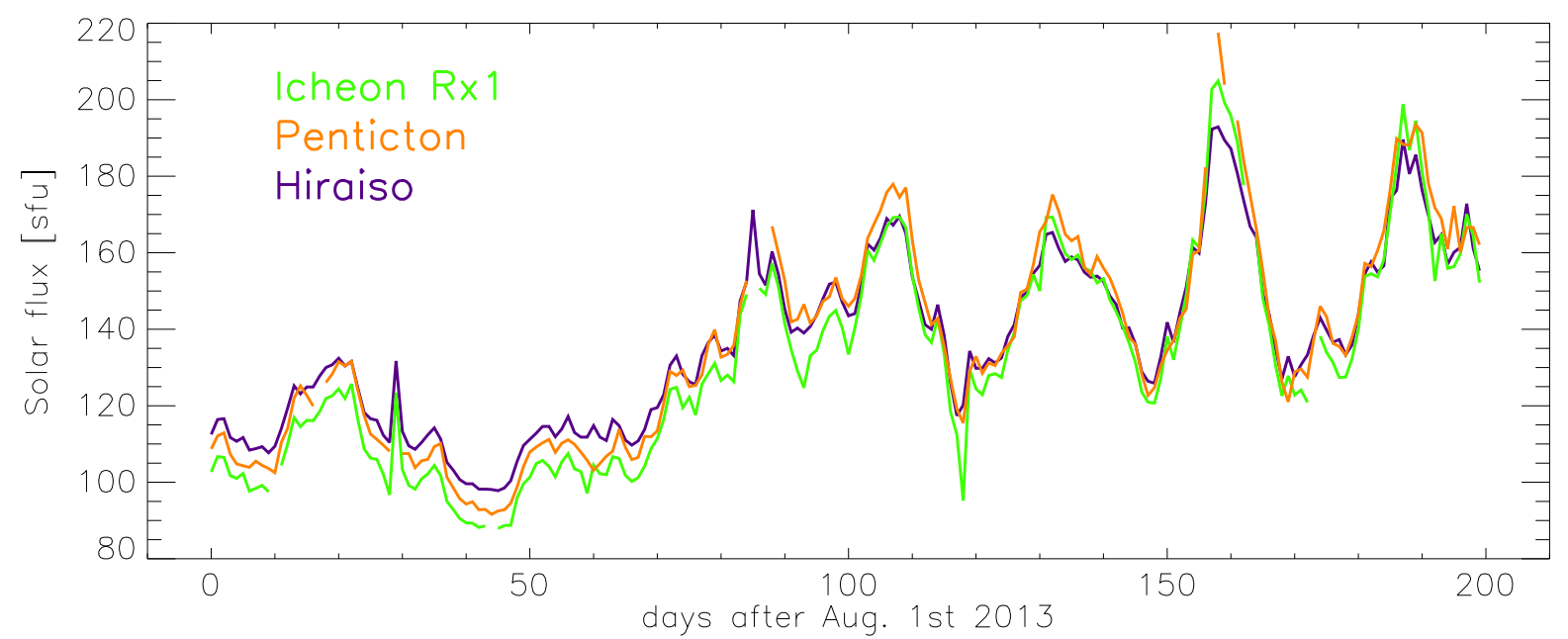

Figure 5. The solar fluxes displayed have been taken in Icheon, Korea, Hiraiso, Japan, and Penticton, Canada from 1 August, 2013 to 16 February, 2014. The Icheon solar fluxes are measured by Rx1. The measurement time was 03:00 UT for both Icheon and Hiraiso, which is seven hours later than that of Canada. Several data points of Icheon Rx1 are missing because of malfunction or bad weather, and we have discarded data from Penticton that are significantly different from the ones obtained by Asian countries due to short-term solar activity.
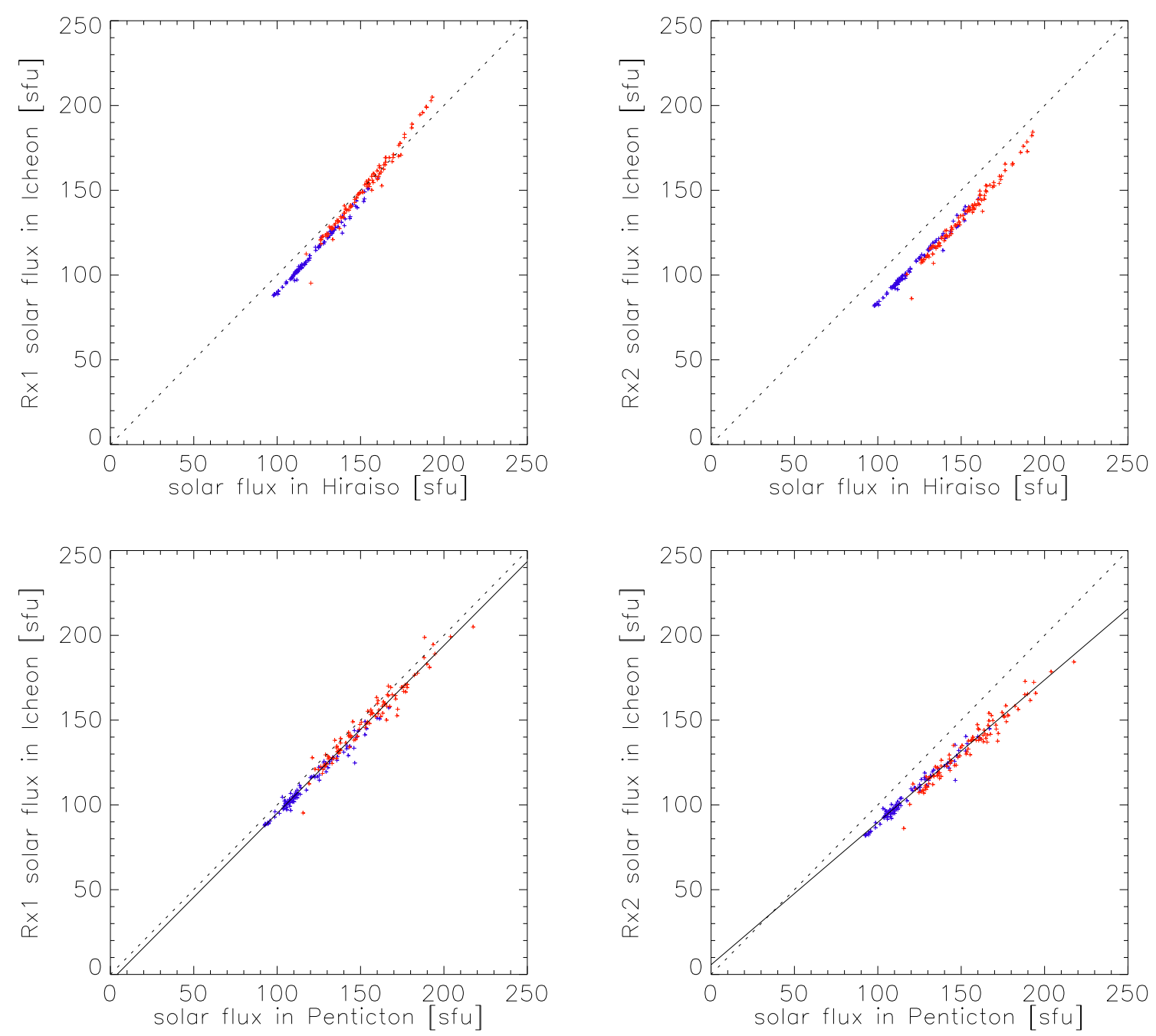

Figure 6. The solar flux in Icheon is compared with that of Penticton, Canada and Hiraiso, Japan from 1 August, 2013 to 16 February, 2014. The blue crosses correspond to data from August to October, 2013, while the red crosses from November 2013 to February 2014. The solid line is the result of the bisector least square fitting. 
In what follows, we will make use of these relationships in converting Icheon Rx1 and Rx2 fluxes to the equivalent Penticton flux. The standard deviations of the converted flux are estimated to be $\sigma=3.2 \mathrm{sfu}$ and $\sigma=3.1$ sfu for Rx1 and Rx2, respectively, but the deviations are mainly due to difference in measurement time between Korea and Canada. Deviations are expected to be smaller as suggested by the smaller scatter between the Icheon and Hiraiso fluxes. The estimated scatter in Hiraiso fluxes is $\sigma=1.9 \mathrm{sfu}$ for Rx2. (We do not estimate the scatter for $\mathrm{Rx} 1$ because of the significant non-linearity of the Hiraiso system.) The intrinsic scatter in the converted Penticton fluxes may be similar to this value. If one is to derive the equivalent Penticton flux from the Icheon data at times other than noon, uncertainty will be greater because of the pointing error.

\section{Conclusion}

We have fabricated solar flux monitors operating at 2.8 $\mathrm{GHz}$ and attached them to the $1.8 \mathrm{~m}$ antenna in Icheon, Korea, to monitor solar radio activity. Control and data acquisition/reduction programs have been developed accordingly. Careful calibration to derive absolute solar flux has been performed with the use of noise sources and termination loads. We have compared the measured flux with that of Penticton, Canada and Hiraiso solar observatory, Japan, to finalize the calibration scheme. We have also derived simple conversion relationships between the measured Icheon flux and the corresponding Penticton flux. Measurement accuracy is found to be better than $\sigma=3.2 \mathrm{sfu}$. We were able to detect several radio bursts, since routine observation was resumed in summer 2013 with the improved calibration accuracy.

\section{ACKNOWLEDGMENTS}

This work is supported by the National Radio Research Agency, Korea.

\section{REFERENCES}

Cerruti, A. P., Kintner, P. M., Gary, D. E., Lanzerotti, L. J., de Paula, E. R., \& Vo, H. B. 2006, Observed Solar Radio Burst Effects on GPS/Wide Area Augmentation System Carrier-to-Noise Ratio, Space Weather, 4, S10006

Gary, D. E., Lanzerotti, L. J., Nita, G. M., \& Thomson, D. J. 2005, Effects of Solar Radio Bursts on Wireless Systems, in Effects of Space Weather on Technology Infrastructure, 176, 203

Isobe, T., Feigelson, E. D., Akritas, M. G., \& Babu, G. J. 1990, Linear Regression in Astronomy, ApJ, 364, 104

Tapping, K. F. 2001, Antenna Calibration Using the 10.7 cm Solar Flux, Workshop on Radar Calibration, Albuquerque, New Mexico, American Meteorological Society

Tapping, K. F., \& Charrois, D. P. 1994, Limits to the Accuracy of the $10.7 \mathrm{~cm}$ Flux, Solar Physics, 150, 305

Tapping, K. F., \& Morton, D. 2013, The Next Generation of Canadian Solar Flux Monitoring, Journal of Physics: Conference Series, 440, 012039

Wilson, T. L., Rohlfs, K., \& Hüttenmeister, S. 2009, Tools of Radio Astronomy (5th edn.) 